\title{
Dynamic fracture of icosahedral model quasicrystals: A molecular dynamics study
}

\author{
Frohmut Rösch, Christoph Rudhart, Johannes Roth, and Hans-Rainer Trebin \\ Institut für Theoretische und Angewandte Physik, \\ Universität Stuttgart, Pfaffenwaldring 57, 70550 Stuttgart, Germany \\ Peter Gumbsch \\ Institut für Zuverlässigkeit von Bauteilen und Systemen, \\ Universität Karlsruhe, Kaiserstr. 12, 76131 Karlsruhe, Germany and \\ Fraunhofer Institut für Werkstoffmechanik, Wöhlerstr. 11, 79108 Freiburg, Germany
}

\begin{abstract}
Ebert et al. [Phys. Rev. Lett. 77, 3827 (1996)] have fractured icosahedral Al-Mn-Pd single crystals in ultrahigh vacuum and have investigated the cleavage planes in-situ by scanning tunneling microscopy (STM). Globular patterns in the STM-images were interpreted as clusters of atoms. These are significant structural units of quasicrystals. The experiments of Ebert et al. imply that they are also stable physical entities, a property controversially discussed currently. For a clarification we performed the first large scale fracture simulations on three-dimensional complex binary systems. We studied the propagation of mode I cracks in an icosahedral model quasicrystal by molecular dynamics techniques at low temperature. In particular we examined how the shape of the cleavage plane is influenced by the clusters inherent in the model and how it depends on the plane structure. Brittle fracture with no indication of dislocation activity is observed. The crack surfaces are rough on the scale of the clusters, but exhibit constant average heights for orientations perpendicular to high symmetry axes. From detailed analyses of the fractured samples we conclude that both, the plane structure and the clusters, strongly influence dynamic fracture in quasicrystals and that the clusters therefore have to be regarded as physical entities.
\end{abstract}

PACS numbers: $62.20 . \mathrm{Mk}, 61.44 . \mathrm{Br}, 02.70 . \mathrm{Ns}$

Keywords: fracture, quasicrystals, molecular dynamics simulations

\section{INTRODUCTION}

Quasicrystals are intermetallic compounds with longrange quasi-periodic translational order. They possess well-defined atomic planes and hence diffract electromagnetic and matter waves into sharp Bragg spots. But they also display atomic clusters as basic building blocks ${ }^{112}$, whose arrangement in space is compatible with the planar structure. These clusters consist for example of several shells of icosahedral symmetry (Bergman-, Mackay-, pseudo-Mackay-clusters). Or they form polytopes, e.g. decagonal prisms, which like the unit cells of periodic crystals fill space, although with large overlaps ("quasi-

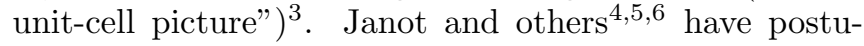
lated that a self-similar hierarchical assembly of the clusters is responsible for the stability of quasicrystals and for many physical properties, like the low electric conductivity. However, it is a controversial and persistent discussion, whether the clusters are merely structural units or whether they represent physical entities. The discussion was fueled by an experiment of Ebert et al ${ }^{17}$, where icosahedral Al-Mn-Pd was fractured under ultrahigh vacuum conditions at room temperature. Scanning tunneling microscopy images of the cleavage planes revealed elements of 0.6 to $1 \mathrm{~nm}$ in diameter ${ }^{8}$. The authors argue that these are the clusters which were circumvented by the crack and hence form highly stable aggregates of matter. Others point out that flat terraces evolve on fivefold surfaces of i-Al-Mn-Pd when annealed at high temperatures $9|10| 11 \mid 12$. As this requires truncated rows of Bergman and Mackay clusters it is stated ${ }^{13}$ that these therefore could not represent firm entities.

In the present article we report on molecular dynamics simulations of crack propagation in a three-dimensional icosahedral model quasicrystal at low temperature. Seed cracks are inserted along different planes and therein along different directions. The fracture planes are carefully analysed to answer the role of clusters in dynamic fracture. In Sec. [II we provide some requirements on the theoretical description of fracture. In Sec. III the model quasicrystal, the molecular dynamics technique, and the methods to visualise the results of the simulations are outlined. Subsequently, in Sec. IV] the simulation results are presented and then discussed in Sec. V]

\section{FRACTURE}

The stress concentration and the strength of the loading at a crack tip are determined by the macroscopic geometry and dimensions of a sample. In linear elastic continuum mechanics, a sharp mode I (opening mode) crack is characterised by a singular stress field and a corresponding displacement field, which both are proportional to the stress intensity factor $K$. This factor is proportional to the applied external load and contains the geometry of the sample. A simple energy based condition for crack propagation is the Griffith criterion 14 . 
It states that a crack is in equilibrium when the change in mechanical energy per unit area of crack advance the energy release rate $G$ - equals the change in surface energy of the two fracture surfaces, $2 \gamma$. In continuum mechanics the energy release rate is proportional to the square of the stress intensity factor for a given mode. A crack then should start moving when the stress intensity factor exceeds the critical Griffith value.

A continuum mechanical description of fracture, however, has a few drawbacks. First, the requirements for linear elasticity are no longer valid near the crack tip where atomic bonds clearly become non-linear and eventually break. Second, a continuum theory neglects the discrete nature of the lattice. Thus, it is fully ignored that fracture of materials is ultimately caused by bond breaking processes on the atomic scale.

A way to understand the processes is to perform numerical experiments, since experimental information on this length scale is difficult to obtain. Molecular dynamics studies have provided useful insight into crack propagation in pure metals and simple intermetallic alloys, whereas in complex metallic alloys the mechanisms are not yet so clear. Atomistic studies show for example that cracks remain stable in a region around the critical stress intensity factor due to the discrete nature of the lattice. This effect is called lattice trapping ${ }^{15}$. A further consequence of the discrete lattice is that the fracture behaviour in one and the same plane can depend on the crack propagation direction 16 . Such observations cannot be explained by a simple continuum mechanical description.

\section{MODEL AND METHOD}

\section{A. Icosahedral binary model}

In the numerical experiments we use a threedimensional model quasicrystal which has been proposed by Henley and Elser ${ }^{17}$ as a structure model for the icosahedral phase of $(\mathrm{Al}, \mathrm{Zn}) \mathrm{Mg}$. This is the simplest possible model quasicrystal that is stabilised by pair potentials. Furthermore it allows Burgers circuit analysis and is a prototype of Bergman-type quasicrystals. As we do not distinguish between $\mathrm{Al}$ and $\mathrm{Zn}$ atoms, we term this decoration icosahedral binary model. It can be obtained by decorating the structure elements of the threedimensional Penrose tiling, the oblate and the prolate rhombohedra (see Fig. 1, top). $\mathrm{Al}$ and $\mathrm{Zn}$ atoms (A atoms) are placed on the vertices and the midpoints of the edges of the rhombohedra. Two Mg atoms (B atoms) divide the long body diagonal of each prolate rhombohedron in ratios $\tau: 1: \tau$, where $\tau$ is the golden mean. Two prolate and two oblate rhombohedra with a common vertex form a rhombic dodecahedron $\frac{18 / 19}{}$. To obtain the icosahedral binary model, in these dodecahedra the atom at the common vertex is removed and the four neighbouring A atoms are transformed into B atoms. Finally these atoms are shifted to the common vertex to divide the edges of the corresponding rhombohedra in a ratio of 1: . Fig. 1 (bottom left) shows the final decoration of these dodecahedra, in which the B atoms form hexagonal bipyramides. This modification increases the number of Bergman-type clusters (see Fig. 1, bottom right) inherent in the structure, leads to a higher stability with the potentials used, and takes better into account the experimentally observed stoichiometry of the quasicrystal. The Bergman-type clusters may also be interpreted as building units of the quasicrystal and are the main feature of the structure apart from the plane structure.

\section{B. Molecular dynamics technique}

As is often done in fracture simulations (see e.g. Abraham ${ }^{20}$ ) we use simple Lennard-Jones potentials to model the interactions. The following facts led to this choice:

First, the very few potentials available for quasicrystals are unsuitable for fracture simulations: These pair potentials stabilise the quasicrystal only for a fixed volume. With free surfaces atoms sometimes simply evaporate (e.g. with potentials based on those of Hafner et al $\left.{ }^{21}\right)$. Very long-ranged potentials with Friedel oscillations (e.g. those of Al-Lehyani et al $\stackrel{[22}{[2}$ ) frequently display such large cohesive energies that nearly no elastic deformation is possible and instead intrinsic cracks develop.

Second, many simulations have proven that model potentials are helping to understand the elementary pro-

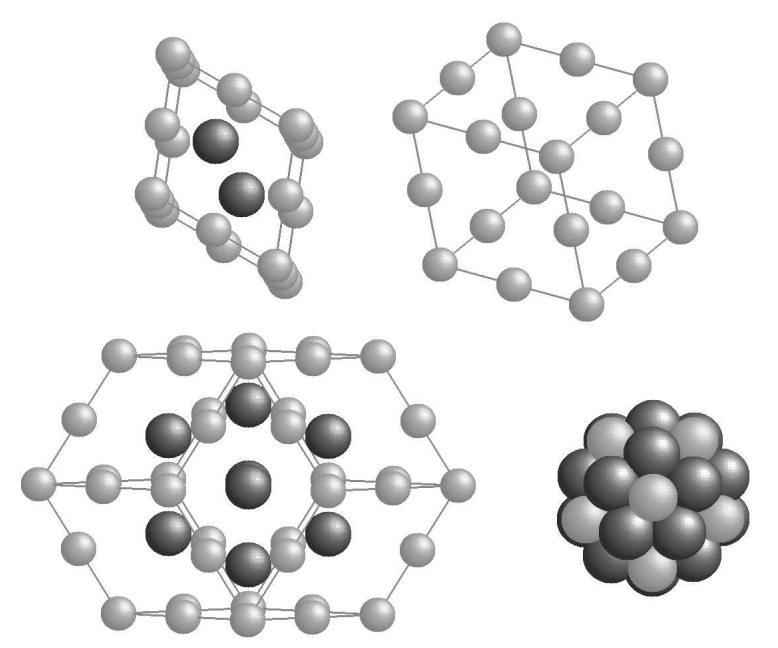

FIG. 1: Tiles of the icosahedral binary model decorated with two types of atoms and the Bergman-type cluster. Top: prolate rhombohedron (left) and oblate rhombohedron (right). Bottom: rhombic dodecahedron (left) and the 45 atoms building the Bergman-type cluster (right) inherent in the model quasicrystal. Small grey and large black spheres denote A and $\mathrm{B}$ atoms respectively. 
cesses in fracture (see e.g. Abraham ${ }^{20}$ ) and so are reasonable when qualitative mechanisms are the centre of interest. For quantitative results, a known limitation is the neglect of non-local and many-body interactions.

Third, the Lennard-Jones potentials used ${ }^{23 \mid 24}$ keep the model stable even under strongest mechanical deformations or irradiation (introduction of point defects) and have been used in our group in many simulations of dislocation motion ${ }^{25}$ or even shock waves ${ }^{26}$. The structure is robust under a wide variation of the potential depths. Very similar potentials have shown to stabilise the icosahedral atomic structure in a simpler mode 27 . It is also known since the early fifties that Lennard-Jones potentials favour icosahedral clusters 28 , indicating that these potentials are useful for structures like icosahedral quasicrystals.

The minima of the potentials for interactions between atoms of the same type are set to $\epsilon_{0}$, whereas the minimum of the potential for the interactions between atoms of different kind is set to $2 \epsilon_{0}$. The conclusions drawn from our simulations, however, remained essentially unchanged if all binding energies are set equal. The shortest distance between two A atoms is denoted $r_{0}$. All masses are set to $m_{0}$. The time is then measured in units of $t_{0}=r_{0} \sqrt{m_{0} / \epsilon_{0}}$.

The molecular dynamics simulations were carried out using the microcanonical ensemble with the program code IMD 29130 . It performs well on a large variety of hardware, including single and dual processor workstations and massively parallel supercomputers.

First, we searched for the potential cleavage planes. According to the Griffith criterion they should be planes of low surface energy ${ }^{31}$. To identify these surfaces we relax a specimen and split it into two parts. Subsequently, the two regions are shifted apart rigidly. The surface energy is then calculated from the energy difference of the artificially cleaved and the undisturbed specimen.

In contrast to simple periodic crystals, the atomistic structure of the planes and therefore also the surface energy in quasicrystals varies strongly within the family of planes perpendicular to a fixed axis. In the present model, surfaces with low interface energy are located perpendicular to two- and fivefold directions at certain heights (Fig. 22). Perpendicular to other directions the plane structure is less pronounced and the minimal surface energies are higher.

Since we are interested in the morphology of fracture surfaces we apply a sample form that allows us to follow the dynamics of the running crack for a long time. For this purpose, a strip geometry is used to model crack propagation with constant energy release rate ${ }^{32}$. The samples consist of about 4 to 5 million atoms, with dimensions of approximately $450 r_{0} \times 150 r_{0} \times 70 r_{0}$. Periodic boundary conditions ${ }^{33}$ are applied in the direction parallel to the crack front. For the other directions, all atoms in the outermost boundary layers of width $2.5 r_{0}$ are held fixed. An atomically sharp seed crack is inserted at a plane of lowest surface energy, from one side to about

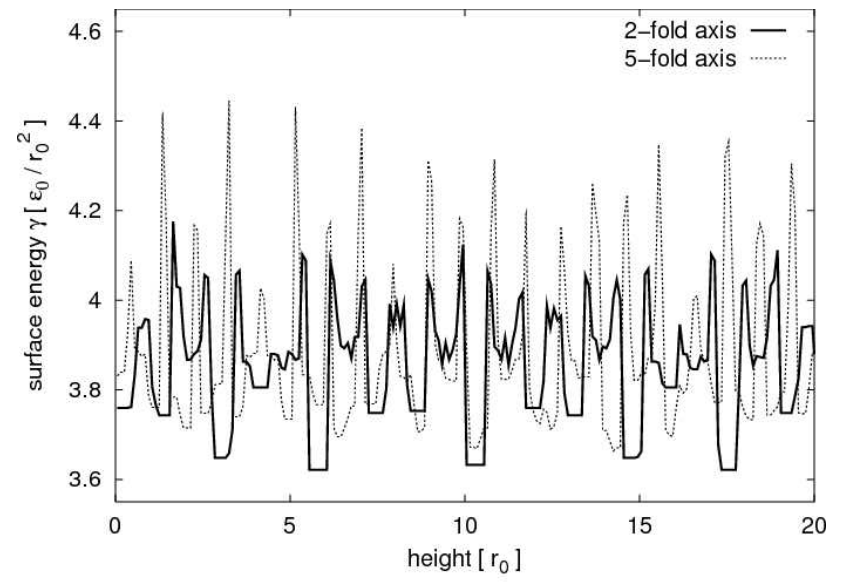

FIG. 2: Surface energy of cleavage planes perpendicular to two- and fivefold axes.

one quarter of the strip length. The system is uniaxially strained perpendicular to the crack plane up to the Griffith load and is relaxed to obtain the displacement field of a stable crack at zero temperature. Then a temperature of about $10^{-4}$ of the melting temperature is applied ${ }^{34}$ to the configurations with and without the relaxed crack. From the resulting configurations we obtain an averaged displacement field for this temperature. The crack now is further loaded by linear scaling of this displacement field. The response of the system then is monitored by molecular dynamics techniques. The sound waves emitted by the propagating crack (see Fig. 3 and online movie ${ }^{35}$ ) are damped away outside of an elliptical stadium ${ }^{32}$ to prevent reflections.

\section{Visualisation}

To study crack propagation on an atomic level the selection and reduction of data is of crucial importance. Due to the large number of atoms required for the study of crack propagation in three-dimensional systems, it is not feasible to write out the positions of all atoms during the simulation, and even less to display all of them. To obtain a first insight into crack propagation only atoms

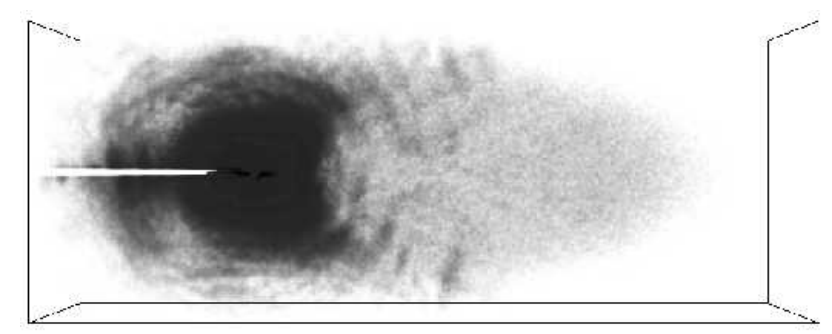

FIG. 3: Kinetic energy density. Sound waves emitted by the propagating crack and the elliptical region without damping are clearly visible. See online movie ${ }^{35}$. 


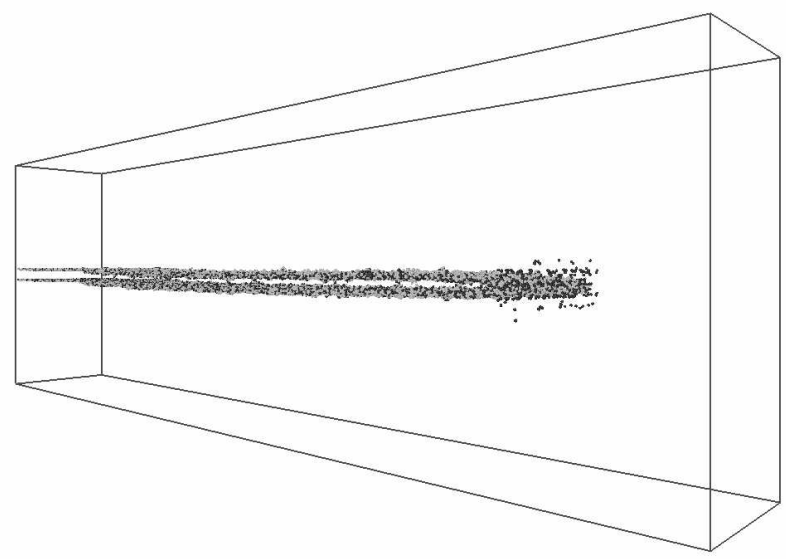

FIG. 4: Snapshot of a simulation with some 4 million atoms. Only atoms with low coordination number are displayed. See online movie 36 .

near the fracture surfaces are of interest. Whereas they can be visualised in periodic crystals by plotting only those atoms whose potential energy exceeds a certain threshold, this technique is not applicable for quasicrystals. Because of the largely varying environments the potential energy varies significantly from atom to atom, even for atoms of the same type in a defect-free sample.

A solution to this problem is to display only those atoms whose coordination number is smaller than a certain threshold. This number is evaluated by counting atoms within the nearest neighbour distance. Like the potential energy, in quasicrystals the coordination number varies from atom to atom, but to a much smaller degree. As atoms near defects have a significantly lower coordination number, it becomes possible to visualise fracture surfaces and dislocation cores. For the A atoms the threshold for the coordination number is set to 12 , whereas for the B atoms it is set to 14 . With this method, the number of atoms to write out or to display can be reduced by three orders of magnitude. Fig. 4 shows a snapshot of a simulation with some 4 million atoms filtered by this technique, which was also used in a movie that is available online 36 .

As can be seen from Fig. 4 the fracture surfaces are rough. Therefore to decide to which fracture surface an atom belongs we apply the displacement vectors between the initial configuration with the built-in seed crack and the fractured sample. The morphology of the fracture planes is then analysed via geometrical scanning of the atoms forming the surfaces. The roughness can be visualised by colour coding the height of the surface in a view normal to the fracture surface.

To investigate the influence of the Bergman-type clusters on cleavage they have to be displayed together with the fracture surfaces intersecting them. This is done by restoring the initial sample without crack at zero temperature. The atoms forming the two sample parts are taken back to their positions in this initial sample and

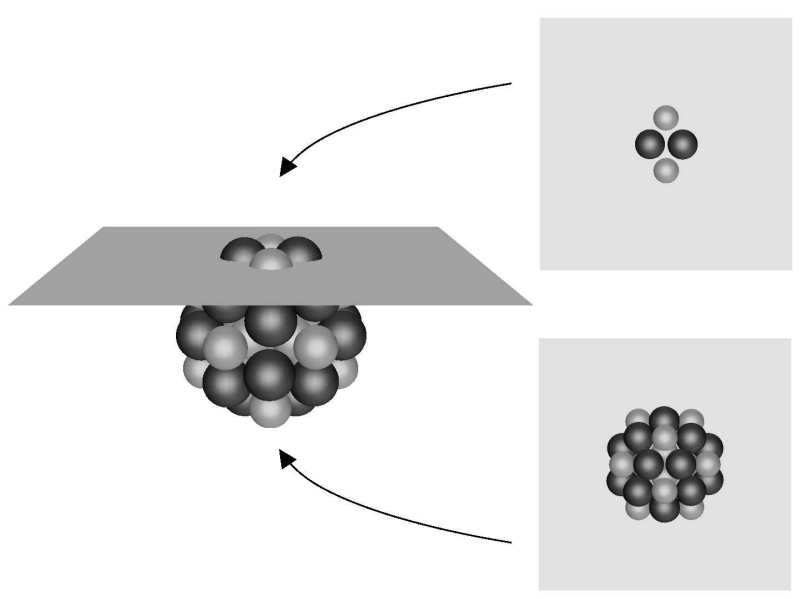

FIG. 5: Bergman-type cluster cut by a flat surface.

then scanned geometrically. In addition all atoms forming clusters in the vicinity of this surfaces are known. By displaying only these atoms and the scanned surface one directly can see where and to which amount clusters are cut. A problem of this kind of visualisation is shown in Fig. 5. where a cluster is cut by a flat surface. When looked-at from above it is obvious that only four atoms are separated from the rest of the cluster. When lookedat from below one could get the impression that the cluster is heavily intersected. On a real fracture surface clusters with centres above and below the crack surface are present. Therefore both views of Fig. 5 are appearing at the same time in a two-dimensional projection. Thus such pictures are not very intuitive.

A way out of this dilemma is presented in Fig. 6. Clusters with midpoints above the crack surface are displayed together with the upper geometrically scanned fracture surface only, the other clusters are shown together with the lower fracture surface. As a result we get two pictures with clusters cut by surfaces. Note that for a qualitative and quantitative analysis always both pictures or sets of data are needed.
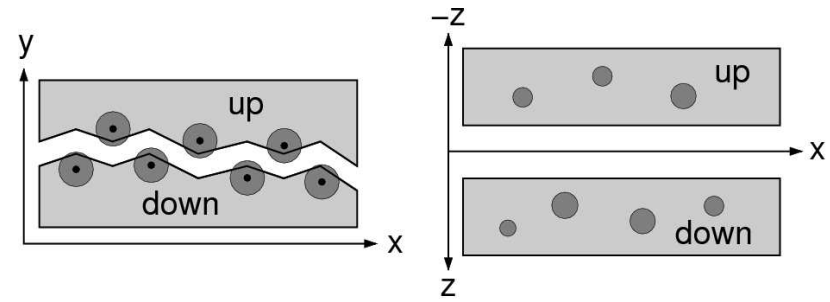

FIG. 6: Visualisation of the clusters cut by the dynamic crack. Midpoints of clusters are indicated as black dots, the clusters are idealised as spheres. The crack propagated from the left to the right. The upper and lower fracture surfaces are projected onto an $x-z$ plane. 


\section{RESULTS}

In this section the results of the numerical simulations are presented. For practical purposes we define $k$ as the stress intensity factor $K$ relative to the stress intensity factor $K_{G}$ at the Griffith load:

$$
k=K / K_{G} .
$$

The orientations of the samples are characterised by the notation $y_{x}$, where $y$ is an axis perpendicular to the cleavage plane and $x$ is an axis in the crack propagation direction (see Fig. 6). An axis perpendicular to a fivefold (5) and a twofold (2) axis is denoted pseudo-twofold (p2) axis.

\section{A. Crack velocities}

Simulations were performed for different orientations with loads in a range from $k=1.1$ to $k=2$ (see notations and loads in Fig. 7). Brittle fracture without any crack tip plasticity is observed irrespective of the orientation of the fracture plane. For loads below $k=1.2$, the crack propagates only a few atomic distances, and then stops. Hence the energy needed for initiating crack propagation is about 1.4 times the value predicted by the Griffith criterion. Therefore, a simple global thermodynamic description of fracture is not applicable. The minimal velocity for brittle crack propagation is about $10 \%$ of the shear wave velocity $v_{s}$. For loads $k \geq 1.2$ the velocity increases monotonically with the applied load. The crack velocities are in a range of $10-45 \%$ of $v_{s}$ (see Fig. 7). At high loads the crack velocities on fivefold cleavage planes show higher average velocities than on the other planes. Velocities for the two different crack propagation directions on the fivefold planes differ significantly at intermediate loads $(k=1.3)$. This coincides with ledges that are produced in the fracture surface (see section IV B and Fig. 8, bottom).

\section{B. Fracture surfaces}

To analyse the morphology of the fracture surfaces, they are displayed as described in section III C. In Fig. 8 the average height is shown in grey, heights above $+2 r_{0}$ are shown in white and heights below $-2 r_{0}$ are shown in black. The crack propagation direction is from the left to the right. The initial fracture surface is flat, as can be seen from the homogeneous regions on the left. The surfaces resulting from the propagation of the crack, however, show pronounced patterns of regions with different heights. From the observation of the fracture surfaces it is already evident that they are rough and that the peak-to-valley roughness is of the order of the diameter of the clusters. The peak-to-valley roughness and the root-mean-square roughness of the height profiles both increase ${ }^{38}$ for higher loads for surfaces without

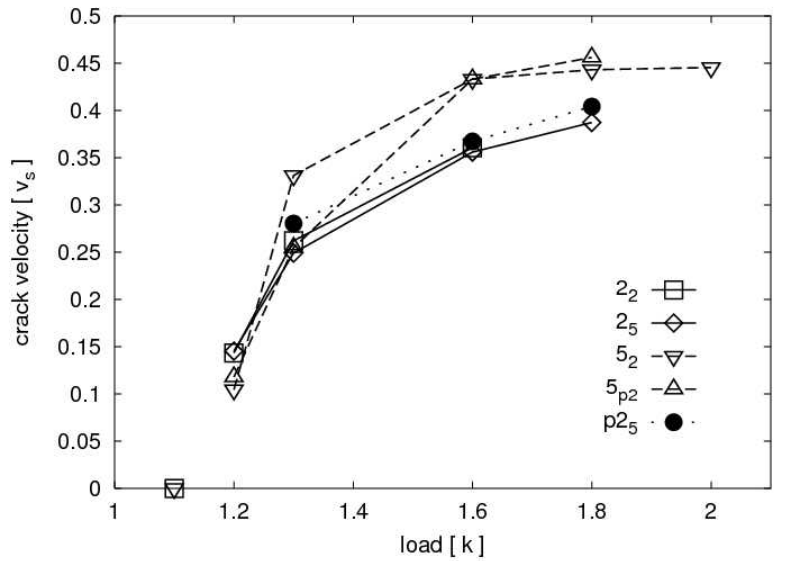

FIG. 7: Average crack velocities for different loads and orientations.

ledge formation. For fracture surfaces perpendicular to twofold and fivefold axes the crack fluctuates about a constant height (in the areas without ledges). In contrast, a crack inserted perpendicular to a pseudo-twofold direction seems to deviate from this plane ${ }^{39}$.

\section{Anisotropy}

As can be seen already from the fracture surfaces perpendicular to a fivefold axis in Fig. 8 crack surfaces for the same cleavage plane differ significantly for different inplane crack propagation directions. For the orientation $5_{\mathrm{p} 2}$ ledges are produced, while no ledges form for the orientation $5_{2}$. By visual inspection of the fracture surfaces (see Fig. 8) and evaluation of height-height-correlation functions $\sqrt{23}$ it becomes evident that for every orientation there are distinct angles in the height profile, which show pronounced height correlations that correspond to markings in the fracture surfaces or to ledges. These angles are given in Table $\mathbb{1}$.

\section{Clusters}

In Fig. 9 the clusters cut by the fracture surfaces are presented as described in section IIIC and Fig. 6. It is obvious from Fig. 9 that the dynamic crack does not perfectly circumvent the clusters, but intersects them partially (right side of Fig. 9). These intersections, however,

TABLE I: Angles observed in the height profiles of the fracture surfaces. Angles measured clockwise from the crack propagation direction get a negative sign.

\begin{tabular}{lccccc}
\hline \hline orientation & $2_{2}$ & $2_{5}$ & $5_{2}$ & $5_{\mathrm{p} 2}$ & $\mathrm{p} 2_{5}$ \\
angles & $0^{\circ}, \pm 32^{\circ}$ & $0^{\circ},+32^{\circ},-58^{\circ}$ & $0^{\circ}, \pm 36^{\circ}$ & $\pm 18^{\circ}$ & $0^{\circ}, \pm 90^{\circ}$ \\
\hline \hline
\end{tabular}



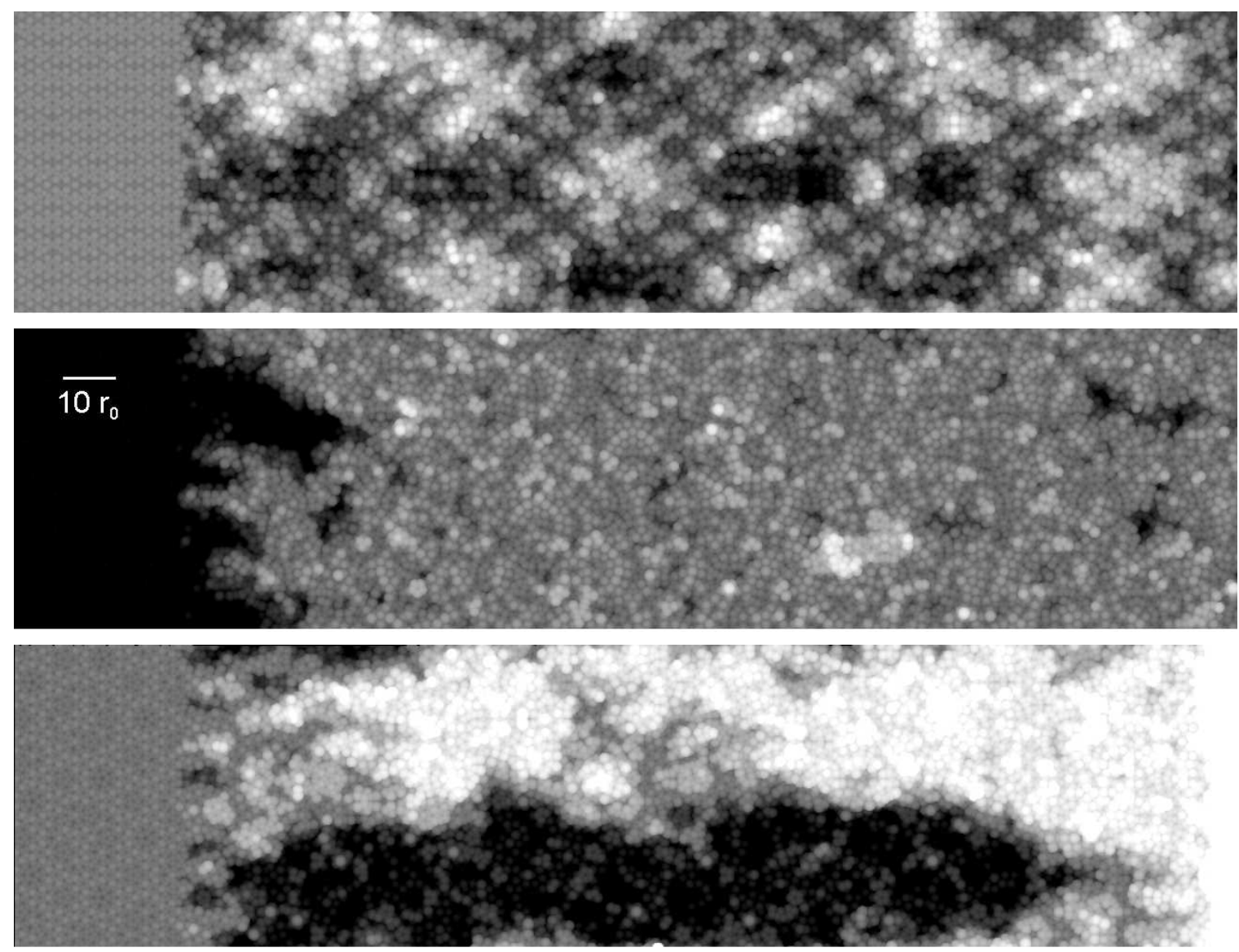

FIG. 8: Height profiles of sections of the simulated fracture surfaces. Load: $k=1.3$, orientation $2_{2}$ (top), $5_{2}$ (middle), $5_{\mathrm{p} 2}$ (bottom). The height increases from black $\left(\leq-2 r_{0}\right)$ to white $\left(\geq+2 r_{0}\right)$. The scanning sphere has the same size as an atom of type B.
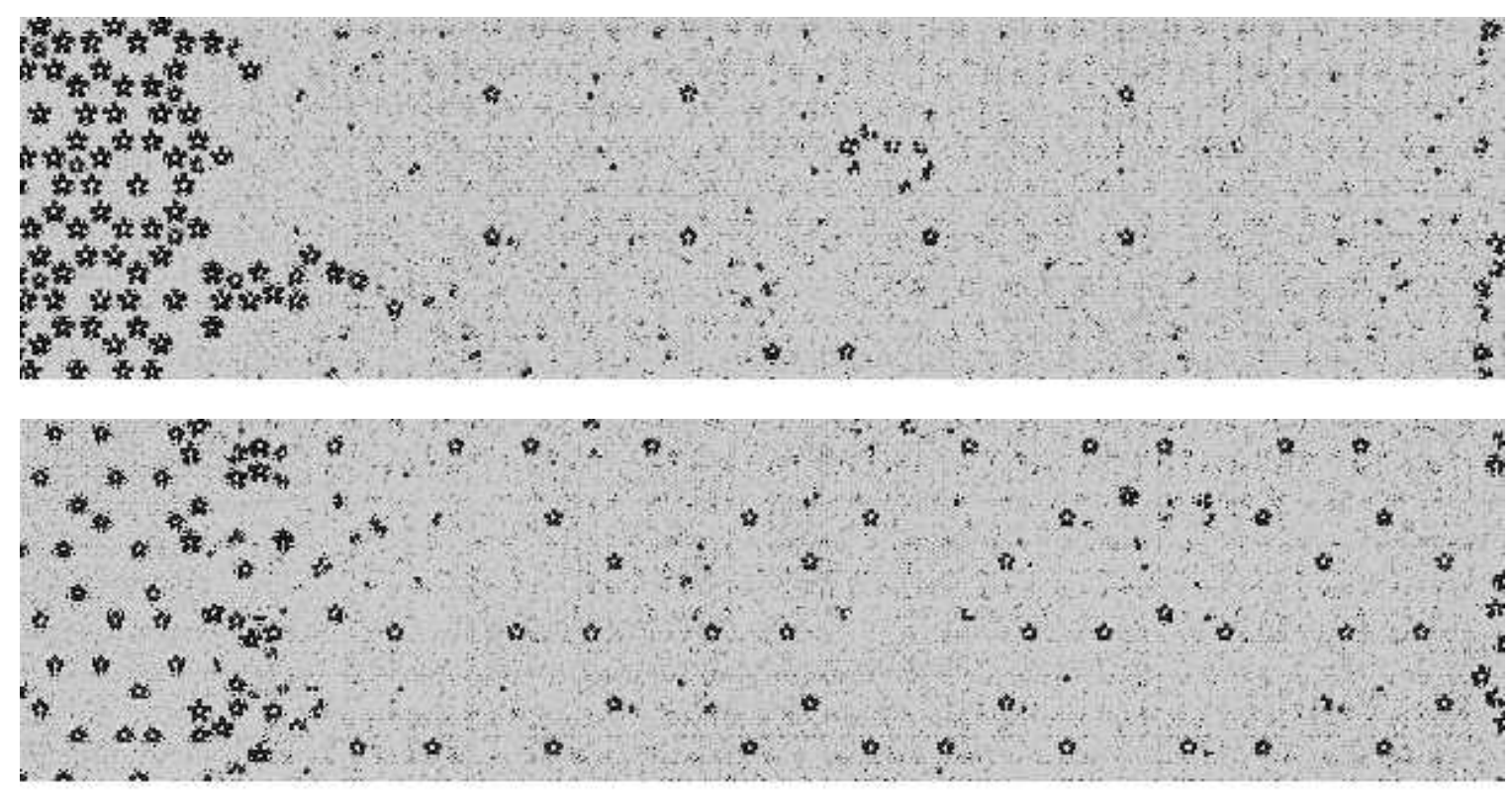

FIG. 9: Clusters cut by the fracture surfaces. The visualisation technique is described in Sec. IIIC and Fig. 6 Load: $k=1.3$, orientation: $5_{2}$. 


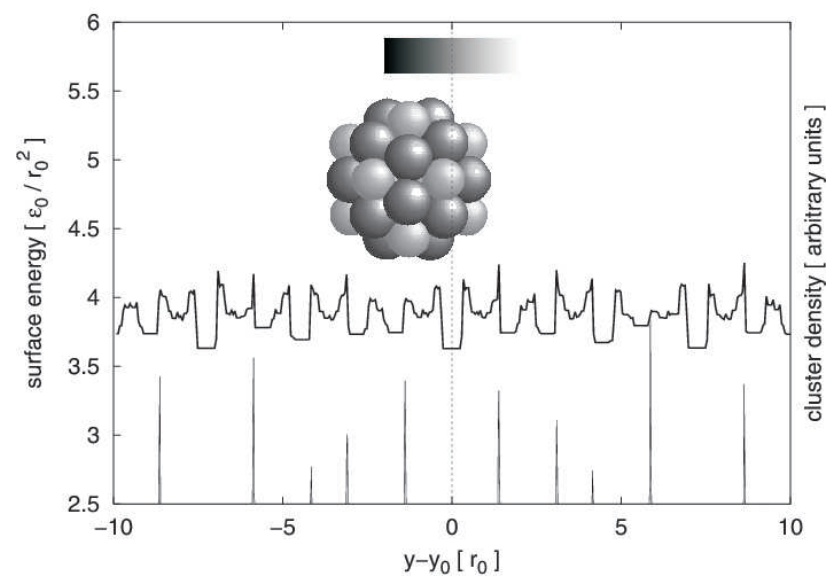

FIG. 10: Surface energy and density of cluster centres for the orientation $2_{2}$. The corresponding fracture surface is shown in Fig. 8, top.

are much less frequent than for the flat seed cracks (left side of Fig. 9). More detailed analyses for different orientations validate this statement. For the orientations perpendicular to twofold and fivefold axes at $k=1.3$ the ratio of clusters cut by the dynamic crack to clusters intersected by flat cuts is approximately 0.6. Additionally the absolute value of clusters cut by the crack for the fivefold surfaces is lower than for the twofold surfaces.

Fig. 10 and Fig. 11 display bottom up: The density of the cluster centres, the surface energy, a cluster in the proper length scale, the grey coding of the heights in Fig. 8 (top, middle), and the position of the seed crack (dashed vertical line). For the twofold fracture surface the low energy seed crack is located between two peaks in the cluster density, whereas for the fivefold surface this seed crack is situated close to a peak of this density. It is evident from the figures that it is not possible to circumvent all clusters by a planar cut.

The grey coding is adjusted to the average height of the fracture surfaces. It is therefore evident from Fig. 11 that the crack deviates for the orientation $5_{2}$ from the low energy cleavage plane of the seed crack to a parallel plane, reducing the number of cluster intersections dramatically (see also Fig. 9 and Fig. 8, middle). Samples cut flat at the new height show slightly higher surface energy (see also Fig. 11). However, for low loads and low roughness the actual fracture surfaces of the dynamic cracks have about $5-15 \%$ higher energies than those of the low energy seed cracks. To assure that the dynamic crack is departing from the initial plane not in a random manner the seed crack was built in at the position colour coded as medium grey in Fig. 11. The resulting fracture surface had a similar roughness but the crack did not change to a parallel plane.

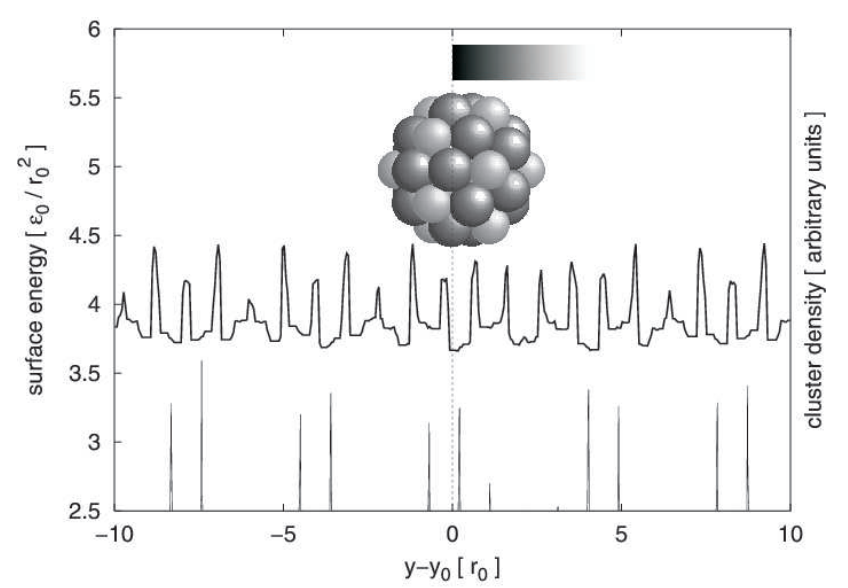

FIG. 11: Surface energy and density of cluster centres for the orientation $5_{2}$. The corresponding fracture surface is shown in Fig. 8 middle.

\section{DISCUSSION}

Taken together the results of our simulations presented above indicate that the distribution of the clusters is crucial for the fracture behaviour: First, circumventing the clusters or intersecting them disturbs the propagating crack and leads to additional radiation. This manifests itself in the crack speed. Dynamic cracks propagating in fivefold planes with few cluster intersections are faster than those in twofold planes, where the absolute number of cluster intersections is higher. Also the generation of ledges for low loads in the orientation $5_{\mathrm{p} 2}$ costs energy and therefore slows down the crack even further. Second, circumvention of the clusters leads to characteristic height-variations. Therefore the peak-tovalley roughness of the fracture surfaces is of the order of the diameter of the clusters. Third, the observed patterns in the fracture surfaces correspond to lines along which the clusters are located. The associated angles are given by the icosahedral symmetry of the sample, namely $0^{\circ}, 18^{\circ}, 31.72^{\circ}, 36^{\circ}, 58.28^{\circ}$, and $90^{\circ}$ (see Table I). Ledges seem to be produced only for the smallest angles measured from the crack propagation direction. Fourth, less clusters are intersected by the fracture surfaces than by the flat seed cracks. Fifth, a seed crack at a low energy cleavage plane deviates to a parallel plane to reduce the number of cluster intersections in spite of the higher energy required to form the fracture surfaces. In contrast, a crack built-in at this new position does not show such a deviation.

Another observation of the simulations is that the plane structure of the quasicrystal also influences fracture. The fracture surfaces that are located perpendicular to the twofold and fivefold symmetry axes show constant average heights.

The three-dimensional quasicrystals give perfect cleavage fracture with no indication of any dislocation activ- 
ity. This is in contrast to results on two-dimensional decagonal quasicrystals, where a dislocation enhanced fracture mechanism has been observed 40 . However a corresponding three-dimensional decagonal quasicrystal would have a periodic direction with a straight dislocation line. In the simulations presented here this direction is also quasiperiodic. As the clusters have a strong influence on fracture they also may bend and hinder dislocation lines. So dislocation emission in the three-dimensional icosahedral quasicrystal modelled here should be less likely than in the two-dimensional decagonal model quasicrystal. Very high stresses are indeed needed to move dislocations in our model quasicrystal in molecular dynamics simulations 25 .

There are also indications for the stability of the clusters from the electronic structure of quasicrystals: First, experiments and ab-initio calculations show that directional bonding may be present within clusters of quasicrystals $41 \mid 42$. Second, the electrons may additionally stabilise the clusters because of their hierarchical structure ${ }^{4}$. Therefore they should be even more stable than we have modelled them with simple pair potentials. With this evidence the results concerning the clusters seem reasonable and should even underestimate their stability.

So far fracture experiments in ultrahigh vacuum have only been performed on icosahedral Al-Mn-Pd, which has a more complicated atomic structure than the icosahedral binary model. Additionally the clusters are not Bergman-type. Therefore we cannot expect to represent this structure on an atomic level, when comparing experiments in Al-Mn-Pd with our simulations. Nevertheless, the size of the clusters, the icosahedral symmetry, and a distinct plane structure are common features and qualitative aspects should be reproduced well, namely the size and shape of the patterns and the appearance of distinct angles on the fracture surfaces. This is indeed the case, as can be seen in Fig. 12. There a geometrically scanned fracture surface generated in our simulations is confronted to an STM-image of Ebert et al.743 at the same length scale. As we were able to correlate the observed structures to the clusters in our model, the similarities corroborate the assumption that the clusters are responsible for the globular structures observed in experiment. More detailed comparisons to fracture experiments in icosahedral Al-Zn-Mg-type quasicrystals would be desirable, but such comparative data is currently not available.

We also performed fracture simulations in a C15 Laves phase with the same Lennard-Jones potentials to give further evidence, that the roughness is correlated to the clusters. This C15 structure is built up from deformed prolate rhombohedra, one of the major tiles of the quasicrystal (see Fig. 1 and Sec. III A. However, no Bergmantype clusters are present. When colour coded like the quasicrystal, fracture surfaces of the C15 cracks lack any roughness at low loads and cleave smoothly on high symmetry planes 44 .

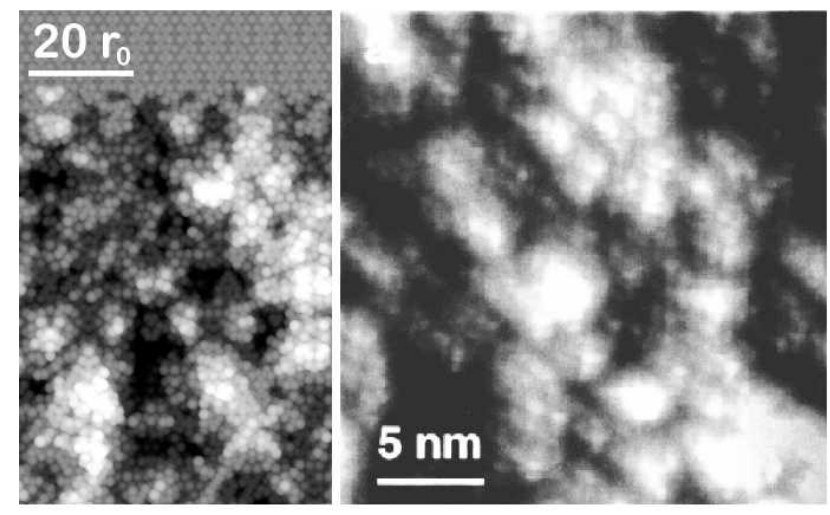

FIG. 12: Fracture surfaces perpendicular to twofold axes. In the left picture (simulation) the atomically sharp seed crack can be seen on the top, whereas below this area the simulated fracture surface appears. The orientation of the sample is $2_{2}$, the load was $k=1.3$. The surface was geometrically scanned with an atom of type B. Thus atomic resolution is achieved. The right picture (experiment, adopted from Ebert et al. ${ }^{43}$ ) shows an STM-image of icosahedral Al-Pd-Mn cleaved in ultrahigh vacuum. As $20 r_{0}$ is approximately $5 \mathrm{~nm}$ the surfaces are displayed at the same length scale.

When a crack traverses a solid, it leaves a typical nonequlibrium surface. Thus, up to this point, we have not dealt with any equilibrium surface. Even the flat Griffith cuts we introduced to perform the numerical experiments are no explicit equilibrium surfaces ${ }^{31}$ by definition. It is known that i-Al-Pd-Mn surfaces sputtered and annealed up to about $900 \mathrm{~K}$ are rough with cluster-like protrusions. Fivefold surfaces annealed at higher temperatures exhibit flat terraces $9|10| 11$, which are believed to be bulk terminated ${ }^{45}$. As fivefold surfaces are not as rough as twofold surfaces, they are often studied in experiment. Adsorption on these surfaces, nevertheless, is often very site-sensitive. A recent review on quasicrystal surfaces was given by McGrath et al.12. All of these observations are consistent with our simulations of non-equilibrium surfaces. Fivefold surfaces experience less roughness than twofold surfaces (also in agreement with the experiments of Ebert et al.7/43, see Fig. 8), where more clusters are intersected. Additionally, since clusters are cut in the simulations, their binding energy is not so large as to term them supermolecules. Thus annealing at very high temperatures can favour flat surfaces 1112 . Nevertheless, selective adsorption may then be related to completion of clusters.

\section{CONCLUSIONS}

We have simulated crack propagation in an icosahedral model quasicrystal. Brittle fracture without any signature of dislocation emission is observed. The fracture surfaces are rough on the scale of the clusters and show constant average heights for orientations perpen- 
dicular to twofold and fivefold axes. Thus both the plane structure and the clusters play an important role in fracture. The influence of the clusters is also seen in the average crack velocities for different orientations, the observed patterns in the fracture surfaces, the anisotropy with respect to the in-plane propagation direction, and the smaller amount of clusters cut by the propagating crack than by planar cuts. The clusters, too, are a reason why the positions of the cleavage planes cannot be predicted by a simple energy criterion. Since partial cluster intersections occur, the binding energy of the clusters is not so large as to term them supermolecules. Nevertheless our observations clearly show that they are not only structural units but physical entities.

\section{Acknowledgments}

Financial support from the Deutsche Forschungsgemeinschaft under contract numbers TR 154/13 and TR $154 / 20-1$ is gratefully acknowledged.
1 C. L. Henley, Phys. Rev. B 43 (1), 993 (1991).

${ }^{2}$ V. Elser, Phil. Mag. B 73 (4), 641 (1996).

3 P. J. Steinhardt, H.-C. Jeong, K. Saitoh, M. Tanaka, E. Abe, and A. P. Tsai, Nature 396 (6706), 55 (1998).

${ }^{4}$ C. Janot and M. de Boissieu, Phys. Rev. Lett. 72 (11), 1674 (1994).

${ }^{5}$ C. Janot, J. Phys.: Condens. Matter 9, 1493 (1997).

6 H.-C. Jeong and P. J. Steinhardt, Phys. Rev. Lett. 73 (14), 1943 (1994).

7 Ph. Ebert, M. Feuerbacher, N. Tamura, M. Wollgarten, and K. Urban, Phys. Rev. Lett. 77 (18), 3827 (1996).

${ }^{8}$ Ph. Ebert, M. Yurechko, F. Kluge, K. Horn, and K. Urban, Quasicrystals - Structure and Physical Properties, WILEY-VCH, ed. H.-R. Trebin, 6.1, 572 (2003).

9 T. M. Schaub, D. E. Bürgler, H.-J. Güntherodt, and J. B. Suck, Phys. Rev. Lett. 73 (9), 1255 (1994).

10 M. Gierer, M. A. Van Hove, A. I. Goldman, Z. Shen, S.-L. Chang, C. J. Jenks, C.-M. Zhang, and P. A. Thiel, Phys. Rev. Lett. 78 (3), 467 (1997).

11 G. Cappello, J. Chevrier, F. Schmithüsen, A. Stierle, V. Formoso, F. Comin, M. de Boissieu, M. Boudard, T. Lograsso, C. Jenks, and D. Delaney, Phys. Rev. B 65, 245405 (2002).

12 R. McGrath, J. Ledieu, E. J. Cox, and R. D. Diehl, J. Phys.: Condens. Matter 14 (4), R119 (2002).

13 G. Kasner, Z. Papadopolos, P. Kramer, and D. E. Bürgler, Quasicrystals - Structure and Physical Properties, WILEY-VCH, ed. H.-R. Trebin, 2.4, 123 (2003).

14 A. A. Griffith, Philos. Trans. R. Soc. Lond. Ser. A 221, 163 (1921).

15 R. Thomson, C. Hsieh, and V. Rana, J. Appl. Phys. 42 (8), 3154 (1971).

${ }^{16}$ P. Gumbsch and R. M. Cannon, Mat. Res. Soc. Bull. 25 (5), 15 (2000).

17 C. L. Henley and V. Elser, Phil. Mag. B 53 (3), L59 (1986).

18 The icosahedral binary model is described and named BIB in J. Roth, Eur. Phys. J. B 15 (1), 7 (2000).

19 In rings of oblate rhombohedra the number of clusters is maximised. No overlapping rhombic dodecahedra are generated. Remaining oblate rhombohedra stay unchanged.

20 F. F. Abraham, Advances in Physics 52 (8), 727 (2003).

21 J. Hafner and M. Krajčí, Europhys. Lett. 13 (4), 335 (1990).

22 I. Al-Lehyani, M. Widom, Y. Wang, N. Moghadam, G. M. Stocks, and J. A. Moriarty, Phys. Rev. B 64, 075109 (2001).

${ }^{23}$ F. Rösch, diploma thesis, Universität Stuttgart (2003), http://elib.uni-stuttgart.de/opus/volltexte/2004/1899/
24 F. Rösch, Ch. Rudhart, P. Gumbsch, and H.-R. Trebin, Mat. Res. Soc. Symp. Proc. 805, LL9.3.1 (2004).

25 G. D. Schaaf, J. Roth, and H.-R. Trebin, Phil. Mag. 83 (21), 2449 (2003).

26 J. Roth, Phys. Rev. B 71, 064102 (2005).

27 J. W. Roth, R. Schilling, and H.-R. Trebin, Phys. Rev. B 51 (22), 15833 (1995).

28 F. C. Frank, Proc. Roy. Soc. Lond. Ser. A 215 (1120), 43 (1952).

29 J. Stadler, R. Mikulla, and H.-R. Trebin, Int. J. Mod. Phys. C 8 (5), 1131 (1997).

30 IMD, the ITAP Molecular Dynamics Program. http://www.itap.physik.uni-stuttgart.de/ imd

31 To simulate fracture with molecular dynamics it is necessary to determine the optimal place for a low energy seed crack and to find out the critical strain for setting the crack into motion. This is always done by determining the energies of flat cuts. Note, that these do not necessarily represent equilibrium surfaces which may be bent and curved.

32 P. Gumbsch, S. J. Zhou, and B. L. Holian, Phys. Rev. B 55 (6), 3445 (1997).

33 As quasicrystals show non-periodic translational order we generate periodic approximants to apply periodic boundary conditions. The 4 to 5 million atoms of our sample then form the unit cell. Because of this high number of atoms the configuration should mechanically behave like the perfect quasicrystal.

34 Here we use equation (2) of Gumbsch et al. ${ }^{32}$ with a "filled stadion damping" $f \equiv 1$.

35 Cracks propagate by breaking bonds between atoms. In this process, energy is dissipated in the form of acoustic waves, as clearly visible in the online movie. The kinetic energy density is colour coded. Regions of high kinetic energy density are shown in red whereas blue indicates areas of lower kinetic energy density.

36 Movie (available online) of a cracking model quasicrystal at a high load $\left(5_{p 2}, k=1.6\right)$. By displaying only atoms with a low coordination number fracture surfaces are visualised. Blue (black in Fig. 1) and red (grey in Fig. 1) balls correspond to the atoms in the icosahedral binary model. Atoms on the border, which are not allowed to move, are coloured in yellow and green. On the left the atomically sharp seed crack can be seen. Due to the high deformation near the crack tip, blue atoms loose their neighbours and show up for short instances.

37 Concerning only the pure phonon term of the elastic energy in linear elastic continuum theory, the icosahedral 
quasicrystal behaves like an isotropic solid.

38 The height profiles were determined via geometrical scanning of the fracture surfaces with an atom of type B at equidistant points separated by $0.2 r_{0}$. For these meshes and e.g. for the orientation $5_{2}$ the root-mean-square roughness increases from $0.39 r_{0}$ at a load of $k=1.3$ (see Fig. 8. middle) to $0.54 r_{0}$ for $k=1.6$. The peak-to-valley roughness increases likewise from $3.7 r_{0}$ to $5.1 r_{0}$. Although the exact values may depend on the stepwidth (resolution), scanning sphere size, and scanned area a general tendency for an increased roughness for increased loads is evident.

39 The question whether this crack finally chooses a plane perpendicular to a twofold or fivefold axis cannot be answered, as simulations with the required sample-size would exceed present computer capabilities.

${ }^{40}$ R. Mikulla, J. Stadler, F. Krul, H.-R. Trebin, and P.
Gumbsch, Phys. Rev. Lett. 81 (15), 3163 (1998).

41 Only clusters in the bulk give reliable information on their relative stability. In ab-initio studies, where the number of atoms is limited, one therefore is restricted to small approximants.

${ }^{42}$ K. Kirihara, T. Nagata, K. Kimura, K. Kato, M. Takata, E. Nishibori, and M. Sakata, Phys. Rev. B 68, 014205 (2003).

43 Ph. Ebert, F. Yue, and K. Urban, Phys. Rev. B 57 (5), 2821 (1998).

44 F. Rösch, P. Gumbsch, and H.-R. Trebin, unpublished.

45 Z. Papadopolos, G. Kasner, J. Ledieu, E. J. Cox, N. V. Richardson, Q. Chen, R. D. Diehl, T. A. Lograsso, A. R. Ross, and R. McGrath, Phys. Rev. B 66, 184207 (2002). 